\title{
A New Method for Inextensible Flows of Timelike Curves in Minkowski Space-Time $\mathbb{E}_{1}^{4}$
}

\author{
Talat Körpinar \\ Department of Mathematics, Muş Alparslan University, 49250 Muş, Turkey \\ Correspondence should be addressed to Talat Körpinar; talatkorpinar@gmail.com
}

Received 3 September 2014; Accepted 19 October 2014; Published 16 December 2014

Academic Editor: Nikolai A. Kudryashov

Copyright (C) 2014 Talat Körpinar. This is an open access article distributed under the Creative Commons Attribution License, which permits unrestricted use, distribution, and reproduction in any medium, provided the original work is properly cited.

We construct a new method for inextensible flows of timelike curves in Minkowski space-time $\mathbb{E}_{1}^{4}$. Using the Frenet frame of the given curve, we present partial differential equations. We give some characterizations for curvatures of a timelike curve in Minkowski space-time $\mathbb{E}_{1}^{4}$.

\section{Introduction}

Numerous processing operations of complex fluids involve free surface deformations; examples include spraying and atomization of fertilizers and pesticides, fiber-spinning operations, paint application, roll-coating of adhesives, and food processing operations such as container- and bottle-filling. Systematically understanding such flows can be extremely difficult because of the large number of different forces that may be involved, including capillarity, viscosity, inertia, gravity, and the additional stresses resulting from the extensional deformation of the microstructure within the fluid. Consequently many free-surface phenomena are described by heuristic and poorly quantified words such as "spinnability," "tackiness," and "stringiness." Additional specialized terms used in other industries include "pituity" in lubricious aqueous coatings, "body" and "length" in the printing ink business, "ropiness" in yogurts, and "long/short textures" in starch processing [1].

The flow of a curve or surface is said to be inextensible if, in the former case, the arc length is preserved, and, in the latter case, if the intrinsic curvature is preserved [2-7]. Physically, inextensible curve and surface flows are characterized by the absence of any strain energy induced from the motion. Kwon investigated inextensible flows of curves and developable surfaces in $R^{3}$. Necessary and sufficient conditions for an inextensible curve flow first are expressed as a partial differential equation involving the curvature and torsion. Then, they derived the corresponding equations for the inextensible flow of a developable surface and showed that it suffices to describe its evolution in terms of two inextensible curve flows [8]. Additionally, there are many works related with inextensible flows [1, 8-15].

In the past two decades, for the need to explain certain physical phenomena and to solve practical problems, geometers and geometric analysis have begun to deal with curves and surfaces which are subject to various forces and which flow or evolve with time in response to those forces so that the metrics are changing. Now, various geometric flows have become one of the central topics in geometric analysis. Many authors have studied geometric flow problems $[1,12,16,17]$.

This study is organised as follows: firstly, we study inextensible flows of timelike curves in Minkowski spacetime. Secondly, using the Frenet frame of the given curve, we present partial differential equations. Finally, we give some characterizations for curvatures of a curve in Minkowski space-time.

\section{Preliminaries}

A "particle" in special relativity means a curve $\alpha$ with a timelike unitary tangent vector $[18,19]$.

Since $g$ is an indefinite metric, recall that a vector $v$ can have one of the following three casual characterizations:

(i) it can be space-like if $g(v, v)>0$ or $v=0$; 
(ii) it can be timelike if $g(v, v)<0$;

(iii) it can be null (light-like) if $g(v, v)=0$ and $v \neq 0$.

Similarly, an arbitrary curve $\alpha$ can be locally space-like, timelike, or null (light-like), if all of its velocity vectors $\alpha^{\prime}$ are, respectively, space-like, timelike, or null. Also, recall that the norm of a vector $v$ is given by

$$
\|v\|=\sqrt{|g(v, v)|} .
$$

Therefore, $v$ is a unit vector if $g(v, v)= \pm 1$. Next, vectors $v, w$ are said to be orthogonal if $g(v, w)=0$ (see [20]). The velocity of the curve $\alpha$ is given by $\left\|\alpha^{\prime}(s)\right\|$.

Denote by $\left\{\mathbf{T}, \mathbf{N}, \mathbf{B}_{1}, \mathbf{B}_{2}\right\}$ the moving Frenet frame along the curve $\alpha(s)$ in the space-time. Then $\mathbf{T}, \mathbf{N}, \mathbf{B}_{1}, \mathbf{B}_{2}$ are, respectively, the tangent, the principal normal, the binormal, and the trinormal vector fields. A space-like or timelike curve $\alpha(s)$ is said to be parameterized by arc length function $s$, if

$$
g\left(\alpha^{\prime}(s), \alpha^{\prime}(s)\right)= \pm 1
$$

Let $\alpha(s)$ be a timelike curve in the space-time, parameterized by arc length function $s$.

The timelike curve $\alpha$ is called timelike Frenet curve if there exist three smooth functions $k_{1}, k_{2}, k_{3}$ on $\alpha$ and smooth nonnull frame field $\left\{\mathbf{T}, \mathbf{N}, \mathbf{B}_{1}, \mathbf{B}_{2}\right\}$ along the curve $\alpha$. Also, the functions $k_{1}, k_{2}$, and $k_{3}$ are called the first, the second, and the third curvature function on $\alpha$, respectively. Then, for the unit speed timelike curve $\alpha$ with nonnull frame vectors [16], the following Frenet equations are given,

$$
\begin{gathered}
\mathbf{T}^{\prime}=k_{1} \mathbf{N}, \\
\mathbf{N}^{\prime}=k_{1} \mathbf{T}+k_{2} \mathbf{B}_{1}, \\
\mathbf{B}_{1}^{\prime}=-k_{2} \mathbf{N}+k_{3} \mathbf{B}_{2}, \\
\mathbf{B}_{2}^{\prime}=-k_{3} \mathbf{B}_{1} .
\end{gathered}
$$

Here, due to characters of Frenet vectors of the timelike curve, $\mathbf{T}, \mathbf{N}, \mathbf{B}_{1}$, and $\mathbf{B}_{2}$ are mutually orthogonal vector fields satisfying equations

$$
\begin{gathered}
g(\mathbf{T}, \mathbf{T})=-1, \\
g(\mathbf{N}, \mathbf{N})=g\left(\mathbf{B}_{1}, \mathbf{B}_{1}\right)=g\left(\mathbf{B}_{2}, \mathbf{B}_{2}\right)=1 .
\end{gathered}
$$

\section{A New Method for Inextensible Flows of Timelike Curves in $\mathbb{E}_{1}^{4}$}

Physically, inextensible curve and surface flows give rise to motions in which no strain energy is induced. The swinging motion of a cord of fixed length or, for example, of a piece of paper carried by the wind, can be described by inextensible curve and surface flows. Such motions arise quite naturally in a wide range of physical applications $[8,11,12]$.

Let $\alpha(u, t)$ be a one parameter family of smooth timelike curves in $\mathbb{E}^{4}$.
Any flow of $\alpha$ can be represented as

$$
\frac{\partial \alpha}{\partial t}=\mathfrak{A}_{1} \mathbf{T}+\mathfrak{A}_{2} \mathbf{N}+\mathfrak{A}_{3} \mathbf{B}_{1}+\mathfrak{A}_{4} \mathbf{B}_{2}
$$

where $\mathfrak{A}_{1}, \mathfrak{\mathfrak { A }}_{2}, \mathfrak{\mathfrak { A }}_{3}, \mathfrak{\mathfrak { A }}_{4}$ are smooth functions.

Let the arc length variation be

$$
s(u, t)=\int_{0}^{u} v d u
$$

In the $\mathbb{E}_{1}^{4}$ the requirement that the curve be not subject to any elongation or compression can be expressed by the condition

$$
\frac{\partial}{\partial t} s(u, t)=\int_{0}^{u} \frac{\partial v}{\partial t} d u=0
$$

Definition 1. The flow $\partial \alpha / \partial t$ in $\mathbb{E}_{1}^{4}$ is said to be inextensible if

$$
\frac{\partial}{\partial t}\left\|\frac{\partial \alpha}{\partial u}\right\|=0
$$

Theorem 2. Let $\partial \alpha / \partial t$ be a smooth flow of $\gamma$. The flow is inextensible if and only if

$$
\frac{\partial \mathfrak{A}_{1}}{\partial u}=-\mathfrak{A}_{2} v k_{1}
$$

Proof. Assume that $\partial \alpha / \partial t$ is inextensible. Then,

$$
\frac{\partial}{\partial t} s(u, t)=\int_{0}^{u} \frac{\partial v}{\partial t} d u=\int_{0}^{u}\left(\frac{\partial \mathfrak{A}_{1}}{\partial u}+\mathfrak{A}_{2} v k_{1}\right) d u=0 .
$$

Substituting (8) in (10) completes the proof of the theorem.

We now restrict ourselves to arc length parameterized curves. That is, $v=1$ and the local coordinate $u$ corresponds to the curve arc length $s$. We require the following lemma.

Lemma 3. If the flow is inextensible, then

$$
\begin{aligned}
\frac{\partial \mathbf{T}}{\partial t}= & \left(\mathfrak{A}_{1} k_{1}+\frac{\partial \mathfrak{A}_{2}}{\partial s}-\mathfrak{A}_{3} k_{2}\right) \mathbf{N} \\
& +\left(\mathfrak{A}_{2} k_{2}+\frac{\partial \mathfrak{A}_{3}}{\partial s}-\mathfrak{A}_{4} k_{3}\right) \mathbf{B}_{1} \\
& +\left(\mathfrak{A}_{3} k_{3}+\frac{\partial \mathfrak{A}_{4}}{\partial s}\right) \mathbf{B}_{2},
\end{aligned}
$$

where $\mathfrak{A}_{1}, \mathfrak{A}_{2}, \mathfrak{\mathfrak { A }}_{3}, \mathfrak{\mathfrak { A }}_{4}$ are smooth functions of time and arc length.

Proof. Using definition of $\alpha$, we have

$$
\begin{aligned}
\frac{\partial}{\partial t} \mathbf{T}= & \left(\frac{\partial \mathfrak{A}_{1}}{\partial s}+\mathfrak{A}_{2} k_{1}\right) \mathbf{T}+\left(\mathfrak{A}_{1} k_{1}+\frac{\partial \mathfrak{A}_{2}}{\partial s}-\mathfrak{A}_{3} k_{2}\right) \mathbf{N} \\
& +\left(\mathfrak{A}_{2} k_{2}+\frac{\partial \mathfrak{A}_{3}}{\partial s}-\mathfrak{A}_{4} k_{3}\right) \mathbf{B}_{1} \\
& +\left(\mathfrak{A}_{3} k_{3}+\frac{\partial \mathfrak{\Re}_{4}}{\partial s}\right) \mathbf{B}_{2} .
\end{aligned}
$$

Substituting (9) in (12), we obtain (11). This completes the proof. 
Now we give the characterization of evolution of first curvature as below.

Theorem 4. Let $\partial \alpha / \partial t$ be inextensible flow of timelike $\alpha$ in $\mathbb{E}_{1}^{4}$. Then, the evolution of $k_{1}$ is given by

$$
\begin{aligned}
\frac{\partial k_{1}}{\partial t}=[ & \frac{\partial}{\partial s}\left(\mathfrak{A}_{1} k_{1}+\frac{\partial \mathfrak{A}_{2}}{\partial s}-\mathfrak{A}_{3} k_{2}\right) \\
& \left.-k_{2}\left(\mathfrak{A}_{2} k_{2}+\frac{\partial \mathfrak{A}_{3}}{\partial s}-\mathfrak{A}_{4} k_{3}\right)\right],
\end{aligned}
$$

where $\mathfrak{A}_{1}, \mathfrak{A}_{2}, \mathfrak{A}_{3}, \mathfrak{\mathfrak { A }}_{4}$ are smooth functions of time and arc length.

Proof. Assume that $\partial \alpha / \partial t$ is inextensible in $\mathbb{E}_{1}^{4}$.

Thus it is easy to obtain that

$$
\begin{aligned}
\frac{\partial}{\partial s} \frac{\partial}{\partial t} \mathbf{T}= & k_{1}\left(\mathfrak{A}_{1} k_{1}+\frac{\partial \mathfrak{A}_{2}}{\partial s}-\mathfrak{A}_{3} k_{2}\right) \mathbf{T} \\
+ & {\left[\frac{\partial}{\partial s}\left(\mathfrak{A}_{1} k_{1}+\frac{\partial \mathfrak{A}_{2}}{\partial s}-\mathfrak{A}_{3} k_{2}\right)\right.} \\
& \left.-k_{2}\left(\mathfrak{A}_{2} k_{2}+\frac{\partial \mathfrak{A}_{3}}{\partial s}-\mathfrak{A}_{4} k_{3}\right)\right] \mathbf{N} \\
+ & {\left[\frac{\partial}{\partial s}\left(\mathfrak{A}_{2} k_{2}+\frac{\partial \mathfrak{A}_{3}}{\partial s}-\mathfrak{A}_{4} k_{3}\right)\right.} \\
& -k_{3}\left(\mathfrak{A}_{3} k_{3}+\frac{\partial \mathfrak{A}_{4}}{\partial s}\right) \\
& \left.+k_{2}\left(\mathfrak{A}_{1} k_{1}+\frac{\partial \mathfrak{A}_{2}}{\partial s}-\mathfrak{A}_{3} k_{2}\right)\right] \mathbf{B}_{1} \\
+ & {\left[\frac{\partial}{\partial s}\left(\mathfrak{A}_{3} k_{3}+\frac{\partial \mathfrak{A}_{4}}{\partial s}\right)\right.} \\
& \left.+k_{3}\left(\mathfrak{A}_{2} k_{2}+\frac{\partial \mathfrak{A}_{3}}{\partial s}-\mathfrak{A}_{4} k_{3}\right)\right] \mathbf{B}_{2} .
\end{aligned}
$$

By the Frenet equations we have

$$
\frac{\partial k_{1}}{\partial t}=\frac{\partial}{\partial t}\left\langle\frac{\partial}{\partial s} \mathbf{T}, \mathbf{N}\right\rangle \text {. }
$$

Also,

$$
\frac{\partial \kappa}{\partial t}=\left\langle\frac{\partial}{\partial t} \frac{\partial}{\partial s} \mathbf{T}, \mathbf{N}\right\rangle+\left\langle\frac{\partial}{\partial s} \mathbf{T}, \frac{\partial}{\partial t} \mathbf{N}\right\rangle
$$

By the definition of flow, we have

$$
\left\langle\mathbf{N}, \frac{\partial}{\partial t} \mathbf{N}\right\rangle=0
$$

Combining these we have

$$
\begin{aligned}
\frac{\partial k_{1}}{\partial t}=[ & \frac{\partial}{\partial s}\left(\mathfrak{A}_{1} k_{1}+\frac{\partial \mathfrak{A}_{2}}{\partial s}-\mathfrak{A}_{3} k_{2}\right) \\
& \left.-k_{2}\left(\mathfrak{A}_{2} k_{2}+\frac{\partial \mathfrak{A}_{3}}{\partial s}-\mathfrak{A}_{4} k_{3}\right)\right] .
\end{aligned}
$$

Thus, we obtain the theorem. This completes the proof.
By this theorem we immediately have the following.

Theorem 5. Consider

$$
\begin{aligned}
\frac{\partial \mathbf{N}}{\partial t}=\left(\mathfrak{A}_{1} k_{1}+\frac{\partial \mathfrak{A}_{2}}{\partial s}-\mathfrak{A}_{3} k_{2}\right) \mathbf{T} \\
+\frac{1}{k_{1}}\left[\frac{\partial}{\partial s}\left(\mathfrak{A}_{2} k_{2}+\frac{\partial \mathfrak{A}_{3}}{\partial s}-\mathfrak{A}_{4} k_{3}\right)\right. \\
-k_{3}\left(\mathfrak{A}_{3} k_{3}+\frac{\partial \mathfrak{A}_{4}}{\partial s}\right) \\
\left.+k_{2}\left(\mathfrak{A}_{1} k_{1}+\frac{\partial \mathfrak{A}_{2}}{\partial s}-\mathfrak{A}_{3} k_{2}\right)\right] \mathbf{B}_{1} \\
+\frac{1}{k_{1}}\left[\frac{\partial}{\partial s}\left(\mathfrak{A}_{3} k_{3}+\frac{\partial \mathfrak{A}_{4}}{\partial s}\right)\right. \\
\left.+k_{3}\left(\mathfrak{A}_{2} k_{2}+\frac{\partial \mathfrak{A}_{3}}{\partial s}-\mathfrak{A}_{4} k_{3}\right)\right] \mathbf{B}_{2},
\end{aligned}
$$

where $\mathfrak{A}_{1}, \mathfrak{A}_{2}, \mathfrak{\mathfrak { A }}_{3}, \mathfrak{\mathfrak { A }}_{4}$ are smooth functions of time and arc length.

Proof. Using Frenet equations, we have

$$
\frac{\partial}{\partial t} \frac{\partial}{\partial s} \mathbf{T}=\frac{\partial k_{1}}{\partial t} \mathbf{N}+k_{1} \frac{\partial \mathbf{N}}{\partial t} .
$$

This implies

$$
\begin{aligned}
\frac{\partial \mathbf{N}}{\partial t}=\left(\mathfrak{A}_{1} k_{1}+\right. & \left.\frac{\partial \mathfrak{A}_{2}}{\partial s}-\mathfrak{A}_{3} k_{2}\right) \mathbf{T} \\
+\frac{1}{k_{1}}[ & \frac{\partial}{\partial s}\left(\mathfrak{A}_{2} k_{2}+\frac{\partial \mathfrak{A}_{3}}{\partial s}-\mathfrak{A}_{4} k_{3}\right) \\
& -k_{3}\left(\mathfrak{A}_{3} k_{3}+\frac{\partial \mathfrak{A}_{4}}{\partial s}\right) \\
& \left.+k_{2}\left(\mathfrak{A}_{1} k_{1}+\frac{\partial \mathfrak{A}_{2}}{\partial s}-\mathfrak{A}_{3} k_{2}\right)\right] \mathbf{B}_{1} \\
+\frac{1}{k_{1}}\left[\frac{\partial}{\partial s}\left(\mathfrak{A}_{3} k_{3}+\frac{\partial \mathfrak{A}_{4}}{\partial s}\right)\right. & \left.+k_{3}\left(\mathfrak{A}_{2} k_{2}+\frac{\partial \mathfrak{A}_{3}}{\partial s}-\mathfrak{A}_{4} k_{3}\right)\right] \mathbf{B}_{2},
\end{aligned}
$$

which completes the proof.

Theorem 6. Let $\partial \alpha / \partial t$ be inextensible flow of $\alpha$ in $\mathbb{E}_{1}^{4}$. Then,

$$
\begin{aligned}
& \frac{\partial \mathbf{B}_{1}}{\partial t} \\
& =\frac{1}{k_{2}}\left[\frac{\partial}{\partial s}\left(\mathfrak{A}_{1} k_{1}+\frac{\partial \mathfrak{A}_{2}}{\partial s}-\mathfrak{A}_{3} k_{2}\right)-\frac{\partial k_{1}}{\partial t}\right] \mathbf{T} \\
& \quad+\frac{1}{k_{2}}\left[\left[k_{1}\left(\mathfrak{A}_{1} k_{1}+\frac{\partial \mathfrak{A}_{2}}{\partial s}-\mathfrak{A}_{3} k_{2}\right)\right.\right.
\end{aligned}
$$




$$
\begin{gathered}
-k_{2}\left[\frac { 1 } { k _ { 1 } } \left[\frac{\partial}{\partial s}\left(\mathfrak{A}_{2} k_{2}+\frac{\partial \mathfrak{A}_{3}}{\partial s}-\mathfrak{A}_{4} k_{3}\right)\right.\right. \\
-k_{3}\left(\mathfrak{A}_{3} k_{3}+\frac{\partial \mathfrak{A}_{4}}{\partial s}\right) \\
\left.+k_{2}\left(\mathfrak{A}_{1} k_{1}+\frac{\partial \mathfrak{A}_{2}}{\partial s}-\mathfrak{A}_{3} k_{2}\right)\right] \\
\left.\left.\left.-k_{1}\left(\mathfrak{A}_{1} k_{1}+\frac{\partial \mathfrak{A}_{2}}{\partial s}-\mathfrak{A}_{3} k_{2}\right)\right]\right]\right] \mathbf{N} \\
+\frac{1}{k_{2}}\left[k _ { 3 } \left[\frac { 1 } { k _ { 1 } } \left[\frac{\partial}{\partial s}\left(\mathfrak{A}_{2} k_{2}+\frac{\partial \mathfrak{A}_{3}}{\partial s}-\mathfrak{A}_{4} k_{3}\right)\right.\right.\right. \\
-k_{3}\left(\mathfrak{A}_{3} k_{3}+\frac{\partial \mathfrak{A}_{4}}{\partial s}\right) \\
\left.\left.+k_{2}\left(\mathfrak{A}_{1} k_{1}+\frac{\partial \mathfrak{A}_{2}}{\partial s}-\mathfrak{A}_{3} k_{2}\right)\right]\right] \\
+\frac{\partial}{\partial s}\left[\frac { 1 } { k _ { 1 } } \left[\frac{\partial}{\partial s}\left(\mathfrak{A}_{3} k_{3}+\frac{\partial \mathfrak{A}_{4}}{\partial s}\right)\right.\right. \\
\left.\left.+k_{3}\left(\mathfrak{A}_{2} k_{2}+\frac{\partial \mathfrak{A}_{3}}{\partial s}-\mathfrak{A}_{4} k_{3}\right)\right]\right] \\
\left.-k_{1}\left(\mathfrak{A}_{3} k_{3}+\frac{\partial \mathfrak{A}_{4}}{\partial s}\right)\right] \mathbf{B}_{2},
\end{gathered}
$$

where $\mathfrak{A}_{1}, \mathfrak{A}_{2}, \mathfrak{A}_{3}, \mathfrak{A}_{4}$ are smooth functions of time and arc length.

Proof. Assume that $\partial \alpha / \partial t$ is inextensible flow of $\alpha$ in $\mathbb{E}^{4}$. Consider

$$
\begin{gathered}
\frac{\partial}{\partial s} \frac{\partial \mathbf{N}}{\partial t} \\
=\frac{\partial}{\partial s}\left(\mathfrak{A}_{1} k_{1}+\frac{\partial \mathfrak{A}_{2}}{\partial s}-\mathfrak{A}_{3} k_{2}\right) \mathbf{T} \\
+\left[\left[k_{1}\left(\mathfrak{A}_{1} k_{1}+\frac{\partial \mathfrak{A}_{2}}{\partial s}-\mathfrak{A}_{3} k_{2}\right)\right.\right. \\
-k_{2}\left[\frac { 1 } { k _ { 1 } } \left[\frac{\partial}{\partial s}\left(\mathfrak{A}_{2} k_{2}+\frac{\partial \mathfrak{A}_{3}}{\partial s}-\mathfrak{A}_{4} k_{3}\right)\right.\right. \\
-k_{3}\left(\mathfrak{A}_{3} k_{3}+\frac{\partial \mathfrak{A}_{4}}{\partial s}\right) \\
\left.\left.\left.\left.+k_{2}\left(\mathfrak{A}_{1} k_{1}+\frac{\partial \mathfrak{A}_{2}}{\partial s}-\mathfrak{A}_{3} k_{2}\right)\right]\right]\right]\right] \mathbf{N} \\
+\left[\frac { \partial } { \partial s } \left[\frac { 1 } { k _ { 1 } } \left[\frac{\partial}{\partial s}\left(\mathfrak{A}_{2} k_{2}+\frac{\partial \mathfrak{A}_{3}}{\partial s}-\mathfrak{A}_{4} k_{3}\right)\right.\right.\right. \\
-k_{3}\left(\mathfrak{A}_{3} k_{3}+\frac{\partial \mathfrak{A}_{4}}{\partial s}\right) \\
\left.\left.+k_{2}\left(\mathfrak{A}_{1} k_{1}+\frac{\partial \mathfrak{A}_{2}}{\partial s}-\mathfrak{A}_{3} k_{2}\right)\right]\right]
\end{gathered}
$$

$$
\begin{aligned}
&-k_{3}\left[\frac{1}{k_{1}}[\right. \frac{\partial}{\partial s}\left(\mathfrak{A}_{3} k_{3}+\frac{\partial \mathfrak{A}_{4}}{\partial s}\right) \\
&\left.\left.\left.+k_{3}\left(\mathfrak{A}_{2} k_{2}+\frac{\partial \mathfrak{A}_{3}}{\partial s}-\mathfrak{A}_{4} k_{3}\right)\right]\right]\right] \mathbf{B}_{1} \\
&+\left[k _ { 3 } \left[\frac { 1 } { k _ { 1 } } \left[\frac{\partial}{\partial s}\left(\mathfrak{A}_{2} k_{2}+\frac{\partial \mathfrak{A}_{3}}{\partial s}-\mathfrak{A}_{4} k_{3}\right)\right.\right.\right. \\
&-k_{3}\left(\mathfrak{A}_{3} k_{3}+\frac{\partial \mathfrak{A}_{4}}{\partial s}\right) \\
&\left.\left.+k_{2}\left(\mathfrak{A}_{1} k_{1}+\frac{\partial \mathfrak{A}_{2}}{\partial s}-\mathfrak{A}_{3} k_{2}\right)\right]\right] \\
&+\frac{\partial}{\partial s}\left[\frac { 1 } { k _ { 1 } } \left[\frac{\partial}{\partial s}\left(\mathfrak{A}_{3} k_{3}+\frac{\partial \mathfrak{A}_{4}}{\partial s}\right)\right.\right. \\
&\left.\left.\left.+k_{3}\left(\mathfrak{A}_{2} k_{2}+\frac{\partial \mathfrak{A}_{3}}{\partial s}-\mathfrak{A}_{4} k_{3}\right)\right]\right]\right] \mathbf{B}_{2} .
\end{aligned}
$$

Thus we compute

$$
\begin{aligned}
\frac{\partial}{\partial t} \frac{\partial \mathbf{N}}{\partial s}= & \frac{\partial k_{1}}{\partial t} \mathbf{T}+\frac{\partial k_{2}}{\partial t} \mathbf{B}_{1}+k_{1}\left(\mathfrak{A}_{1} k_{1}+\frac{\partial \mathfrak{A}_{2}}{\partial s}-\mathfrak{A}_{3} k_{2}\right) \mathbf{N} \\
& +k_{1}\left(\mathfrak{A}_{2} k_{2}+\frac{\partial \mathfrak{A}_{3}}{\partial s}-\mathfrak{A}_{4} k_{3}\right) \mathbf{B}_{1} \\
& +k_{1}\left(\mathfrak{A}_{3} k_{3}+\frac{\partial \mathfrak{A}_{4}}{\partial s}\right) \mathbf{B}_{2}+k_{2} \frac{\partial}{\partial t} \mathbf{B}_{1} .
\end{aligned}
$$

Then we can easily see that

$$
\begin{gathered}
k_{2} \frac{\partial \mathbf{B}_{1}}{\partial t} \quad\left[\frac{\partial}{\partial s}\left(\mathfrak{A}_{1} k_{1}+\frac{\partial \mathfrak{A}_{2}}{\partial s}-\mathfrak{A}_{3} k_{2}\right)-\frac{\partial k_{1}}{\partial t}\right] \mathbf{T} \\
+\left[\left[k_{1}\left(\mathfrak{A}_{1} k_{1}+\frac{\partial \mathfrak{A}_{2}}{\partial s}-\mathfrak{A}_{3} k_{2}\right)\right.\right. \\
-k_{2}\left[\frac { 1 } { k _ { 1 } } \left[\frac{\partial}{\partial s}\left(\mathfrak{A}_{2} k_{2}+\frac{\partial \mathfrak{A}_{3}}{\partial s}-\mathfrak{A}_{4} k_{3}\right)\right.\right. \\
-k_{3}\left(\mathfrak{A}_{3} k_{3}+\frac{\partial \mathfrak{A}_{4}}{\partial s}\right) \\
\left.+k_{2}\left(\mathfrak{A}_{1} k_{1}+\frac{\partial \mathfrak{A}_{2}}{\partial s}-\mathfrak{A}_{3} k_{2}\right)\right] \\
\left.\left.\left.+k_{1}\left(\mathfrak{A}_{1} k_{1}+\frac{\partial \mathfrak{A}_{2}}{\partial s}-\mathfrak{A}_{3} k_{2}\right)\right]\right]\right] \mathbf{N} \\
+\left[\frac { \partial } { \partial s } \left[\frac{\partial}{k_{1}}\left(\mathfrak{\mathfrak { A }}_{2} k_{2}+\frac{\partial \mathfrak{A}_{3}}{\partial s}-\mathfrak{A}_{4} k_{3}\right)\right.\right. \\
-k_{3}\left(\mathfrak{A}_{3} k_{3}+\frac{\partial \mathfrak{A}_{4}}{\partial s}\right) \\
\left.\left.+k_{2}\left(\mathfrak{A}_{1} k_{1}+\frac{\partial \mathfrak{A}_{2}}{\partial s}-\mathfrak{A}_{3} k_{2}\right)\right]\right]
\end{gathered}
$$




$$
\begin{gathered}
-k_{3}\left[\frac { 1 } { k _ { 1 } } \left[\frac{\partial}{\partial s}\left(\mathfrak{A}_{3} k_{3}+\frac{\partial \mathfrak{A}_{4}}{\partial s}\right)\right.\right. \\
\left.\left.+k_{3}\left(\mathfrak{A}_{2} k_{2}+\frac{\partial \mathfrak{A}_{3}}{\partial s}-\mathfrak{A}_{4} k_{3}\right)\right]\right] \\
\left.-k_{1}\left(\mathfrak{A}_{2} k_{2}+\frac{\partial \mathfrak{A}_{3}}{\partial s}-\mathfrak{A}_{4} k_{3}\right)\right] \mathbf{B}_{1} \\
+\left[k _ { 3 } \left[\frac { 1 } { k _ { 1 } } \left[\frac{\partial}{\partial s}\left(\mathfrak{A}_{2} k_{2}+\frac{\partial \mathfrak{A}_{3}}{\partial s}-\mathfrak{A}_{4} k_{3}\right)\right.\right.\right. \\
-k_{3}\left(\mathfrak{A}_{3} k_{3}+\frac{\partial \mathfrak{A}_{4}}{\partial s}\right) \\
\left.\left.+k_{2}\left(\mathfrak{A}_{1} k_{1}+\frac{\partial \mathfrak{A}_{2}}{\partial s}-\mathfrak{A}_{3} k_{2}\right)\right]\right] \\
+\frac{\partial}{\partial s}\left[\frac { 1 } { k _ { 1 } } \left[\frac{\partial}{\partial s}\left(\mathfrak{A}_{3} k_{3}+\frac{\partial \mathfrak{A}_{4}}{\partial s}\right)\right.\right. \\
\left.\left.+k_{3}\left(\mathfrak{A}_{2} k_{2}+\frac{\partial \mathfrak{A}_{3}}{\partial s}-\mathfrak{A}_{4} k_{3}\right)\right]\right] \\
\left.+k_{1}\left(\mathfrak{A}_{3} k_{3}+\frac{\partial \mathfrak{A}_{4}}{\partial s}\right)\right] \mathbf{B}_{2} .
\end{gathered}
$$

From definition of flow, we have

$$
\left\langle\mathbf{B}_{1}, \frac{\partial}{\partial t} \mathbf{B}_{1}\right\rangle=0 .
$$

Thus, we obtain the theorem. The proof of theorem is completed.

Now we give the characterization of evolution of second curvature as below.

Theorem 7. The evolution of $k_{2}$ is given by

$$
\begin{gathered}
\frac{\partial k_{2}}{\partial t}=\frac{\partial}{\partial s}\left[\frac { 1 } { k _ { 1 } } \left[\frac{\partial}{\partial s}\left(\mathfrak{A}_{2} k_{2}+\frac{\partial \mathfrak{A}_{3}}{\partial s}-\mathfrak{A}_{4} k_{3}\right)\right.\right. \\
-k_{3}\left(\mathfrak{A}_{3} k_{3}+\frac{\partial \mathfrak{A}_{4}}{\partial s}\right) \\
\left.\left.+k_{2}\left(\mathfrak{A}_{1} k_{1}+\frac{\partial \mathfrak{A}_{2}}{\partial s}-\mathfrak{A}_{3} k_{2}\right)\right]\right] \\
-k_{3}\left[\frac { 1 } { k _ { 1 } } \left[\frac{\partial}{\partial s}\left(\mathfrak{A}_{3} k_{3}+\frac{\partial \mathfrak{A}_{4}}{\partial s}\right)\right.\right. \\
\left.\left.\left.\left.+k_{3}\left(\mathfrak{A}_{2} k_{2}+\frac{\partial \mathfrak{A}_{3}}{\partial s}-\mathfrak{A}_{4} k_{3}\right)\right]\right]\right]\right] \\
-k_{1}\left(\mathfrak{A}_{2} k_{2}+\frac{\partial \mathfrak{A}_{3}}{\partial s}-\mathfrak{A}_{4} k_{3}\right),
\end{gathered}
$$

where $\mathfrak{A}_{1}, \mathfrak{A}_{2}, \mathfrak{A}_{3}, \mathfrak{A}_{4}$ are smooth functions of time and arc length.

Proof. It is obvious from Theorem 6. This completes the proof.
Theorem 8. Let $\partial \alpha / \partial t$ be inextensible flow of $\alpha$ in $\mathbb{E}_{1}^{4}$. Then,

$$
\frac{\partial \mathbf{B}_{2}}{\partial t}
$$$$
=\frac{1}{k_{3}}\left[\frac{\partial}{\partial s}\left[\frac{1}{k_{2}}\left[\frac{\partial}{\partial s}\left(\mathfrak{A}_{1} k_{1}+\frac{\partial \mathfrak{A}_{2}}{\partial s}-\mathfrak{A}_{3} k_{2}\right)-\frac{\partial k_{1}}{\partial t}\right]\right]\right.
$$$$
+\left[\frac { 1 } { k _ { 2 } } \left[\left[k_{1}\left(\mathfrak{A}_{1} k_{1}+\frac{\partial \mathfrak{A}_{2}}{\partial s}-\mathfrak{A}_{3} k_{2}\right)\right.\right.\right.
$$$$
-k_{2}\left[\frac { 1 } { k _ { 1 } } \left[\frac{\partial}{\partial s}\left(\mathfrak{A}_{2} k_{2}+\frac{\partial \mathfrak{A}_{3}}{\partial s}-\mathfrak{A}_{4} k_{3}\right)\right.\right.
$$$$
-k_{3}\left(\mathfrak{A}_{3} k_{3}+\frac{\partial \mathfrak{A}_{4}}{\partial s}\right)
$$$$
\left.+k_{2}\left(\mathfrak{A}_{1} k_{1}+\frac{\partial \mathfrak{A}_{2}}{\partial s}-\mathfrak{A}_{3} k_{2}\right)\right]
$$$$
\left.\left.-k_{1}\left(\mathfrak{A}_{1} k_{1}+\frac{\partial \mathfrak{A}_{2}}{\partial s}-\mathfrak{A}_{3} k_{2}\right)\right]\right] k_{1}
$$$$
\left.\left.\left.+k_{2}\left(\mathfrak{A}_{1} k_{1}+\frac{\partial \mathfrak{A}_{2}}{\partial s}-\mathfrak{A}_{3} k_{2}\right)\right]\right]\right] \mathrm{T}
$$$$
+\frac{1}{k_{3}}\left[\left[\frac { 1 } { k _ { 2 } } \left[\frac{\partial}{\partial s}\left(\mathfrak{A}_{1} k_{1}+\frac{\partial \mathfrak{A}_{2}}{\partial s}-\mathfrak{A}_{3} k_{2}\right)\right.\right.\right.
$$$$
\left.\left.-\frac{\partial k_{1}}{\partial t}\right]\right] k_{1}
$$$$
+\frac{\partial}{\partial s}\left[\frac { 1 } { k _ { 2 } } \left[\left[k_{1}\left(\mathfrak{A}_{1} k_{1}+\frac{\partial \mathfrak{A}_{2}}{\partial s}-\mathfrak{A}_{3} k_{2}\right)\right.\right.\right.
$$$$
-k_{2}\left[\frac { 1 } { k _ { 1 } } \left[\frac{\partial}{\partial s}\left(\mathfrak{A}_{2} k_{2}+\frac{\partial \mathfrak{A}_{3}}{\partial s}-\mathfrak{A}_{4} k_{3}\right)\right.\right.
$$$$
-k_{3}\left(\mathfrak{A}_{3} k_{3}+\frac{\partial \mathfrak{A}_{4}}{\partial s}\right)
$$$$
\left.+k_{2}\left(\mathfrak{A}_{1} k_{1}+\frac{\partial \mathfrak{A}_{2}}{\partial s}-\mathfrak{A}_{3} k_{2}\right)\right]
$$$$
\left.\left.-k_{1}\left(\mathfrak{A}_{1} k_{1}+\frac{\partial \mathfrak{A}_{2}}{\partial s}-\mathfrak{A}_{3} k_{2}\right)\right]\right]
$$$$
\left.\left.\left.+\frac{\partial k_{2}}{\partial t}\right]\right]\right] \mathbf{N}
$$$$
+\frac{1}{k_{3}}\left[[ \frac { 1 } { k _ { 2 } } ] \left[k_{1}\left(\mathfrak{A}_{1} k_{1}+\frac{\partial \mathfrak{A}_{2}}{\partial s}-\mathfrak{A}_{3} k_{2}\right)\right.\right.
$$$$
-k_{2}\left[\frac { 1 } { k _ { 1 } } \left[\frac{\partial}{\partial s}\left(\mathfrak{A}_{2} k_{2}+\frac{\partial \mathfrak{A}_{3}}{\partial s}-\mathfrak{A}_{4} k_{3}\right)\right.\right.
$$$$
-k_{3}\left(\mathfrak{A}_{3} k_{3}+\frac{\partial \mathfrak{A}_{4}}{\partial s}\right)
$$$$
\left.+k_{2}\left(\mathfrak{A}_{1} k_{1}+\frac{\partial \mathfrak{A}_{2}}{\partial s}-\mathfrak{A}_{3} k_{2}\right)\right]
$$$$
\left.\left.-k_{1}\left(\mathfrak{A}_{1} k_{1}+\frac{\partial \mathfrak{A}_{2}}{\partial s}-\mathfrak{A}_{3} k_{2}\right)\right]\right] k_{2}
$$ 


$$
\begin{aligned}
& -k_{3}\left[\frac { 1 } { k _ { 2 } } \left[k _ { 3 } \left[\frac { 1 } { k _ { 1 } } \left[\frac{\partial}{\partial s}\left(\mathfrak{\mathfrak { A }}_{2} k_{2}+\frac{\partial \mathfrak{A}_{3}}{\partial s}-\mathfrak{A}_{4} k_{3}\right)\right.\right.\right.\right. \\
& -k_{3}\left(\mathfrak{\mathfrak { A }}_{3} k_{3}+\frac{\partial \mathfrak{\mathfrak { A }}_{4}}{\partial s}\right) \\
& \left.\left.+k_{2}\left(\mathfrak{A}_{1} k_{1}+\frac{\partial \mathfrak{A}_{2}}{\partial s}-\mathfrak{A}_{3} k_{2}\right)\right]\right] \\
& +\frac{\partial}{\partial s}\left[\frac { 1 } { k _ { 1 } } \left[\frac{\partial}{\partial s}\left(\mathfrak{A}_{3} k_{3}+\frac{\partial \mathfrak{A}_{4}}{\partial s}\right)\right.\right. \\
& +k_{3}\left(\mathfrak{A}_{2} k_{2}+\frac{\partial \mathfrak{A}_{3}}{\partial s}\right. \\
& \left.\left.\left.-\mathfrak{A}_{4} k_{3}\right)\right]\right] \\
& \left.\left.-k_{1}\left(\mathfrak{A}_{3} k_{3}+\frac{\partial \mathfrak{\mathscr { A }}_{4}}{\partial s}\right)\right]\right] \\
& +\frac{k_{2}}{k_{1}}\left[\frac{\partial}{\partial s}\left(\mathfrak{A}_{2} k_{2}+\frac{\partial \mathfrak{A}_{3}}{\partial s}-\mathfrak{A}_{4} k_{3}\right)\right. \\
& -k_{3}\left(\mathfrak{A}_{3} k_{3}+\frac{\partial \mathfrak{A}_{4}}{\partial s}\right) \\
& \left.\left.\left.\left.+k_{2}\left(\mathfrak{A}_{1} k_{1}+\frac{\partial \mathfrak{U}_{2}}{\partial s}-\mathfrak{A}_{3} k_{2}\right)\right]\right]\right]\right] \mathbf{B}_{1}
\end{aligned}
$$

where $\mathfrak{A}_{1}, \mathfrak{A}_{2}, \mathfrak{\mathfrak { A }}_{3}, \mathfrak{\mathfrak { A }}_{4}$ are smooth functions of time and arc length.

Proof. Differentiating (22) with respect to $s$,

$$
\begin{gathered}
\frac{\partial}{\partial s} \frac{\partial \mathbf{B}_{1}}{\partial t} \\
=\left[\frac{\partial}{\partial s}\left[\frac{1}{k_{2}}\left[\frac{\partial}{\partial s}\left(\mathfrak{A}_{1} k_{1}+\frac{\partial \mathfrak{A}_{2}}{\partial s}-\mathfrak{A}_{3} k_{2}\right)-\frac{\partial k_{1}}{\partial t}\right]\right]\right. \\
+\left[\frac { 1 } { k _ { 2 } } \left[\left[k_{1}\left(\mathfrak{A}_{1} k_{1}+\frac{\partial \mathfrak{A}_{2}}{\partial s}-\mathfrak{A}_{3} k_{2}\right)\right.\right.\right. \\
-k_{2}\left[\frac { 1 } { k _ { 1 } } \left[\frac{\partial}{\partial s}\left(\mathfrak{A}_{2} k_{2}+\frac{\partial \mathfrak{A}_{3}}{\partial s}-\mathfrak{A}_{4} k_{3}\right)\right.\right. \\
+k_{3}\left(\mathfrak{A}_{3} k_{3}+\frac{\partial \mathfrak{A}_{4}}{\partial s}\right) \\
+\left[\frac{1}{k_{2}}\left[\frac{\partial}{\partial s}\left(\mathfrak{A}_{1} k_{1}+\frac{\partial \mathfrak{A}_{2}}{\partial s}-\mathfrak{A}_{3} k_{2}\right)-\frac{\partial \mathfrak{A}_{2}}{\partial s}-\mathfrak{A}_{3} k_{2}\right)\right] \\
+\frac{1}{k_{2}}\left[\left[k_{1}\left(\mathfrak{A}_{1} k_{1}+\frac{\partial \mathfrak{A}_{2}}{\partial s}-\mathfrak{A}_{3} k_{2}\right)\right.\right. \\
\left.\left.\left.\left.+k_{1}\left(\mathfrak{A}_{1} k_{1}+\frac{\partial \mathfrak{A}_{2}}{\partial s}-\mathfrak{A}_{3} k_{2}\right)\right]\right] k_{1}\right]\right] \mathbf{T}
\end{gathered}
$$

$$
\begin{aligned}
& -k_{2}\left[\frac { 1 } { k _ { 1 } } \left[\frac{\partial}{\partial s}\left(\mathfrak{\mathfrak { A }}_{2} k_{2}+\frac{\partial \mathfrak{A}_{3}}{\partial s}-\mathfrak{A}_{4} k_{3}\right)\right.\right. \\
& -k_{3}\left(\mathfrak{\mathfrak { A }}_{3} k_{3}+\frac{\partial \mathfrak{A}_{4}}{\partial s}\right) \\
& \left.+k_{2}\left(\mathfrak{A}_{1} k_{1}+\frac{\partial \mathfrak{A}_{2}}{\partial s}-\mathfrak{A}_{3} k_{2}\right)\right] \\
& \left.\left.\left.\left.-k_{1}\left(\mathfrak{A}_{1} k_{1}+\frac{\partial \mathfrak{P}_{2}}{\partial s}-\mathfrak{A}_{3} k_{2}\right)\right]\right]\right]\right] \mathbf{N} \\
& +\left[\left[\frac { 1 } { k _ { 2 } } \left[\left[k_{1}\left(\mathfrak{A}_{1} k_{1}+\frac{\partial \mathfrak{A}_{2}}{\partial s}-\mathfrak{A}_{3} k_{2}\right)\right.\right.\right.\right. \\
& -k_{2}\left[\frac { 1 } { k _ { 1 } } \left[\frac{\partial}{\partial s}\left(\mathfrak{\mathfrak { A }}_{2} k_{2}+\frac{\partial \mathfrak{A}_{3}}{\partial s}-\mathfrak{A}_{4} k_{3}\right)\right.\right. \\
& -k_{3}\left(\mathfrak{A}_{3} k_{3}+\frac{\partial \mathfrak{A}_{4}}{\partial s}\right) \\
& \left.+k_{2}\left(\mathfrak{A}_{1} k_{1}+\frac{\partial \mathfrak{U}_{2}}{\partial s}-\mathfrak{A}_{3} k_{2}\right)\right] \\
& \left.\left.-k_{1}\left(\mathfrak{A}_{1} k_{1}+\frac{\partial \mathfrak{\mathfrak { A }}_{2}}{\partial s}-\mathfrak{\mathfrak { A }}_{3} k_{2}\right)\right]\right] k_{2} \\
& -k_{3}\left[\frac { 1 } { k _ { 2 } } \left[k _ { 3 } \left[\frac { 1 } { k _ { 1 } } \left[\frac{\partial}{\partial s}\left(\mathfrak{A}_{2} k_{2}+\frac{\partial \mathfrak{A}_{3}}{\partial s}-\mathfrak{A}_{4} k_{3}\right)\right.\right.\right.\right. \\
& -k_{3}\left(\mathfrak{\mathfrak { A }}_{3} k_{3}+\frac{\partial \mathfrak{\mathcal { A }}_{4}}{\partial s}\right) \\
& \left.\left.+k_{2}\left(\mathfrak{A}_{1} k_{1}+\frac{\partial \mathfrak{\mathfrak { A }}_{2}}{\partial s}-\mathfrak{\mathfrak { A }}_{3} k_{2}\right)\right]\right] \\
& +\frac{\partial}{\partial s}\left[\frac { 1 } { k _ { 1 } } \left[\frac{\partial}{\partial s}\left(\mathfrak{A}_{3} k_{3}+\frac{\partial \mathfrak{A}_{4}}{\partial s}\right)\right.\right. \\
& \left.\left.+k_{3}\left(\mathfrak{A}_{2} k_{2}+\frac{\partial \mathfrak{A}_{3}}{\partial s}-\mathfrak{A}_{4} k_{3}\right)\right]\right] \\
& \left.\left.\left.\left.\left.-k_{1}\left(\mathfrak{A}_{3} k_{3}+\frac{\partial \mathfrak{A}_{4}}{\partial s}\right)\right]\right]\right]\right]\right] \mathbf{B}_{1} \\
& +\frac{\partial}{\partial s}\left[\frac { 1 } { k _ { 2 } } \left[k _ { 3 } \left[\frac { 1 } { k _ { 1 } } \left[\frac{\partial}{\partial s}\left(\mathfrak{A}_{2} k_{2}+\frac{\partial \mathfrak{A}_{3}}{\partial s}-\mathfrak{A}_{4} k_{3}\right)\right.\right.\right.\right. \\
& -k_{3}\left(\mathfrak{\Re}_{3} k_{3}+\frac{\partial \mathfrak{\Re}_{4}}{\partial s}\right) \\
& \left.\left.+k_{2}\left(\mathfrak{A}_{1} k_{1}+\frac{\partial \mathfrak{A}_{2}}{\partial s}-\mathfrak{A}_{3} k_{2}\right)\right]\right] \\
& +\frac{\partial}{\partial s}\left[\frac { 1 } { k _ { 1 } } \left[\frac{\partial}{\partial s}\left(\mathfrak{A}_{3} k_{3}+\frac{\partial \mathfrak{A}_{4}}{\partial s}\right)\right.\right. \\
& \left.\left.+k_{3}\left(\mathfrak{A}_{2} k_{2}+\frac{\partial \mathfrak{A}_{3}}{\partial s}-\mathfrak{A}_{4} k_{3}\right)\right]\right] \\
& \left.\left.-k_{1}\left(\mathfrak{A}_{3} k_{3}+\frac{\partial \mathfrak{\Re}_{4}}{\partial s}\right)\right]\right] \mathbf{B}_{2} .
\end{aligned}
$$


Thus we easily obtain that

$$
\begin{gathered}
\frac{\partial}{\partial t} \frac{\partial \mathbf{B}_{1}}{\partial s}=k_{3} \frac{\partial \mathbf{B}_{2}}{\partial t}-k_{2}\left(\mathfrak{A}_{1} k_{1}+\frac{\partial \mathfrak{A}_{2}}{\partial s}-\mathfrak{A}_{3} k_{2}\right) \mathbf{T}-\frac{\partial k_{2}}{\partial t} \mathbf{N} \\
-\frac{k_{2}}{k_{1}}\left[\frac{\partial}{\partial s}\left(\mathfrak{\Re}_{2} k_{2}+\frac{\partial \mathfrak{A}_{3}}{\partial s}-\mathfrak{A}_{4} k_{3}\right)\right. \\
-k_{3}\left(\mathfrak{A}_{3} k_{3}+\frac{\partial \mathfrak{A}_{4}}{\partial s}\right) \\
\left.+k_{2}\left(\mathfrak{A}_{1} k_{1}+\frac{\partial \mathfrak{A}_{2}}{\partial s}-\mathfrak{A}_{3} k_{2}\right)\right] \mathbf{B}_{1} \\
+\left[-\frac{k_{2}}{k_{1}}\left[\frac{\partial}{\partial s}\left(\mathfrak{A}_{3} k_{3}+\frac{\partial \mathfrak{A}_{4}}{\partial s}\right)\right.\right. \\
\left.+k_{3}\left(\mathfrak{A}_{2} k_{2}+\frac{\partial \mathfrak{A}_{3}}{\partial s}-\mathfrak{A}_{4} k_{3}\right)\right] \\
\left.+\frac{\partial k_{3}}{\partial t}\right] \mathbf{B}_{2} .
\end{gathered}
$$

Hence, the proof is complete.

Now we give the characterization of evolution of third curvature as below.

Theorem 9. If the flow is inextensible, then

$$
\begin{gathered}
\frac{\partial k_{3}}{\partial t}=\frac{k_{2}}{k_{1}}\left[\frac{\partial}{\partial s}\left(\mathfrak{A}_{3} k_{3}+\frac{\partial \mathfrak{A}_{4}}{\partial s}\right)+k_{3}\left(\mathfrak{A}_{2} k_{2}+\frac{\partial \mathfrak{A}_{3}}{\partial s}-\mathfrak{A}_{4} k_{3}\right)\right] \\
+\frac{\partial}{\partial s}\left[\frac { 1 } { k _ { 2 } } \left[k _ { 3 } \left[\frac { 1 } { k _ { 1 } } \left[\frac{\partial}{\partial s}\left(\mathfrak{A}_{2} k_{2}+\frac{\partial \mathfrak{A}_{3}}{\partial s}-\mathfrak{A}_{4} k_{3}\right)\right.\right.\right.\right. \\
-k_{3}\left(\mathfrak{\mathfrak { A }}_{3} k_{3}+\frac{\partial \mathfrak{A}_{4}}{\partial s}\right) \\
\left.\left.+k_{2}\left(\mathfrak{A}_{1} k_{1}+\frac{\partial \mathfrak{A}_{2}}{\partial s}-\mathfrak{A}_{3} k_{2}\right)\right]\right] \\
+\frac{\partial}{\partial s}\left[\frac { 1 } { k _ { 1 } } \left[\frac{\partial}{\partial s}\left(\mathfrak{A}_{3} k_{3}+\frac{\partial \mathfrak{A}_{4}}{\partial s}\right)\right.\right. \\
\left.\left.+k_{3}\left(\mathfrak{A}_{2} k_{2}+\frac{\partial \mathfrak{A}_{3}}{\partial s}-\mathfrak{A}_{4} k_{3}\right)\right]\right] \\
\left.\left.-k_{1}\left(\mathfrak{A}_{3} k_{3}+\frac{\partial \mathfrak{A}_{4}}{\partial s}\right)\right]\right]
\end{gathered}
$$

where $\mathfrak{A}_{1}, \mathfrak{\mathfrak { A }}_{2}, \mathfrak{\mathfrak { A }}_{3}, \mathfrak{\mathfrak { A }}_{4}$ are smooth functions of time and arc length.

Proof. It is obvious from Theorem 8. This completes the proof.

\section{Conflict of Interests}

The author declares that there is no conflict of interests regarding the publication of this paper.

\section{References}

[1] G. H. McKinley, Dimensionless Groups for Understanding Free Surface Flows of Complex Fluids.

[2] U. Abresch and J. Langer, "The normalized curve shortening flow and homothetic solutions," Journal of Differential Geometry, vol. 23, no. 2, pp. 175-196, 1986.

[3] S. J. Altschuler and M. A. Grayson, Shortening Space Curves and Flow through Singularities, vol. 823 of IMA Preprint Series, Institute for Mathematics and Its Applications, 1991.

[4] B. Andrews, "Evolving convex curves," Calculus of Variations and Partial Differential Equations, vol. 7, no. 4, pp. 315-371, 1998.

[5] J. H. Caltenco, R. Linares, and J. L. López-Bonilla, "Intrinsic geometry of curves and the Lorentz equation," Czechoslovak Journal of Physics, vol. 52, no. 7, pp. 839-842, 2002.

[6] M. Carmeli, "Motion of a charge in a gravitational field," Physical Review B, vol. 138, pp. B1003-B1007, 1965.

[7] P. Crouch and F. Silva Leite, "The dynamic interpolation problem: on Riemannian manifolds, Lie groups, and symmetric spaces," Journal of Dynamical and Control Systems, vol. 1, no. 2, pp. 177-202, 1995.

[8] D. Y. Kwon, F. C. Park, and D. P. Chi, "Inextensible flows of curves and developable surfaces," Applied Mathematics Letters, vol. 18, no. 10, pp. 1156-1162, 2005.

[9] M. Ergüt, E. Turhan, and T. Körpınar, "Characterization of inextensible flows of spacelike curves with sabban frame in $S_{1}^{2}$," Boletim da Sociedade Paranaense de Matematica, vol. 31, no. 2, pp. 47-53, 2013.

[10] M. Gage and R. S. Hamilton, "The heat equation shrinking convex plane curves," Journal of Differential Geometry, vol. 23, no. 1, pp. 69-96, 1986.

[11] M. A. Grayson, "The heat equation shrinks embedded plane curves to round points," Journal of Differential Geometry, vol. 26, no. 2, pp. 285-314, 1987.

[12] T. Körpinar and E. Turhan, "A new version of inextensible flows of spacelike curves with timelike $\mathrm{B}_{2}$ in Minkowski space-time $E_{1}^{4,}$ Differential Equations and Dynamical Systems, vol. 21, no. 3, pp. 281-290, 2013.

[13] H. Q. Lu, J. S. Todhunter, and T. W. Sze, "Congruence conditions for nonplanar developable surfaces and their application to surface recognition," CVGIP: Image Understanding, vol. 58, no. 3, pp. 265-285, 1993.

[14] L. Ma and D. Chen, "Curve shortening in a Riemannian manifold," Annali di Matematica Pura ed Applicata, vol. 186, no. 4, pp. 663-684, 2007.

[15] E. Turhan and T. Körpinar, "On characterization of timelike horizontal biharmonic curves in the lorentzian heisenberg group Heis ${ }^{3}$," Zeitschrift fur Naturforschung A: Journal of Physical Sciences, vol. 65, no. 8, pp. 641-648, 2010.

[16] Z.-S. Shea, X. Chena, and F. Hussaina, "A lie-group derivation of a multi-layer mixing length formula for turbulent channel and pipe flow," In press, http://arxiv.org/abs/1112.6312.

[17] D. C. Wilcox, Turbulence Modeling for CFD, DCW Industries, 2006.

[18] A. Einstein, Relativity: The Special and General Theory, Henry Holt, New York, NY, USA, 1920.

[19] B. O'Neill, Semi-Riemannian Geometry with Applications to Relativity, Academic Press, 1983.

[20] M. P. do Carmo, Differential Geometry of Curves and Surfaces, Prentice-Hall, Englewood Cliffs, NJ, USA, 1976. 


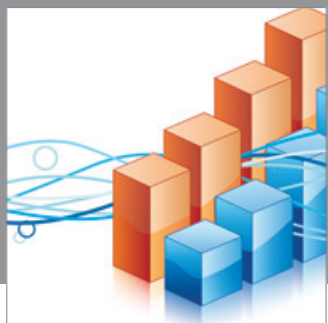

Advances in

Operations Research

mansans

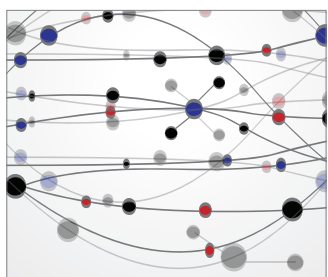

The Scientific World Journal
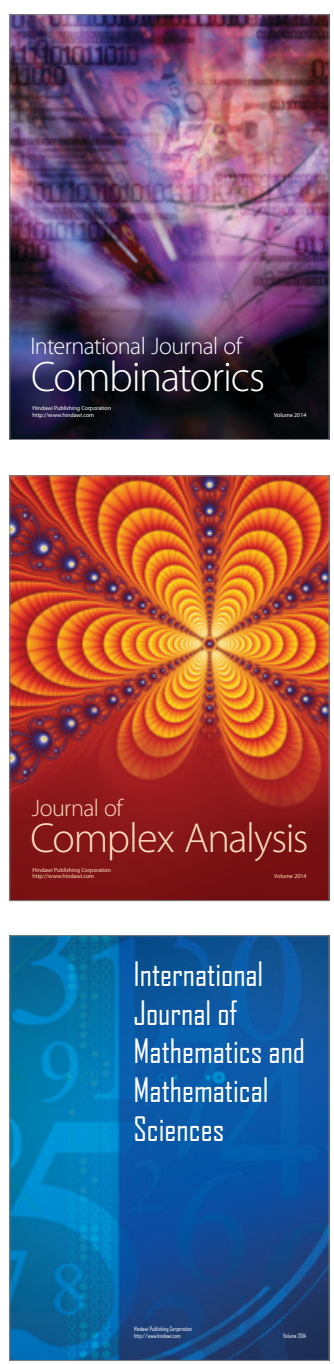
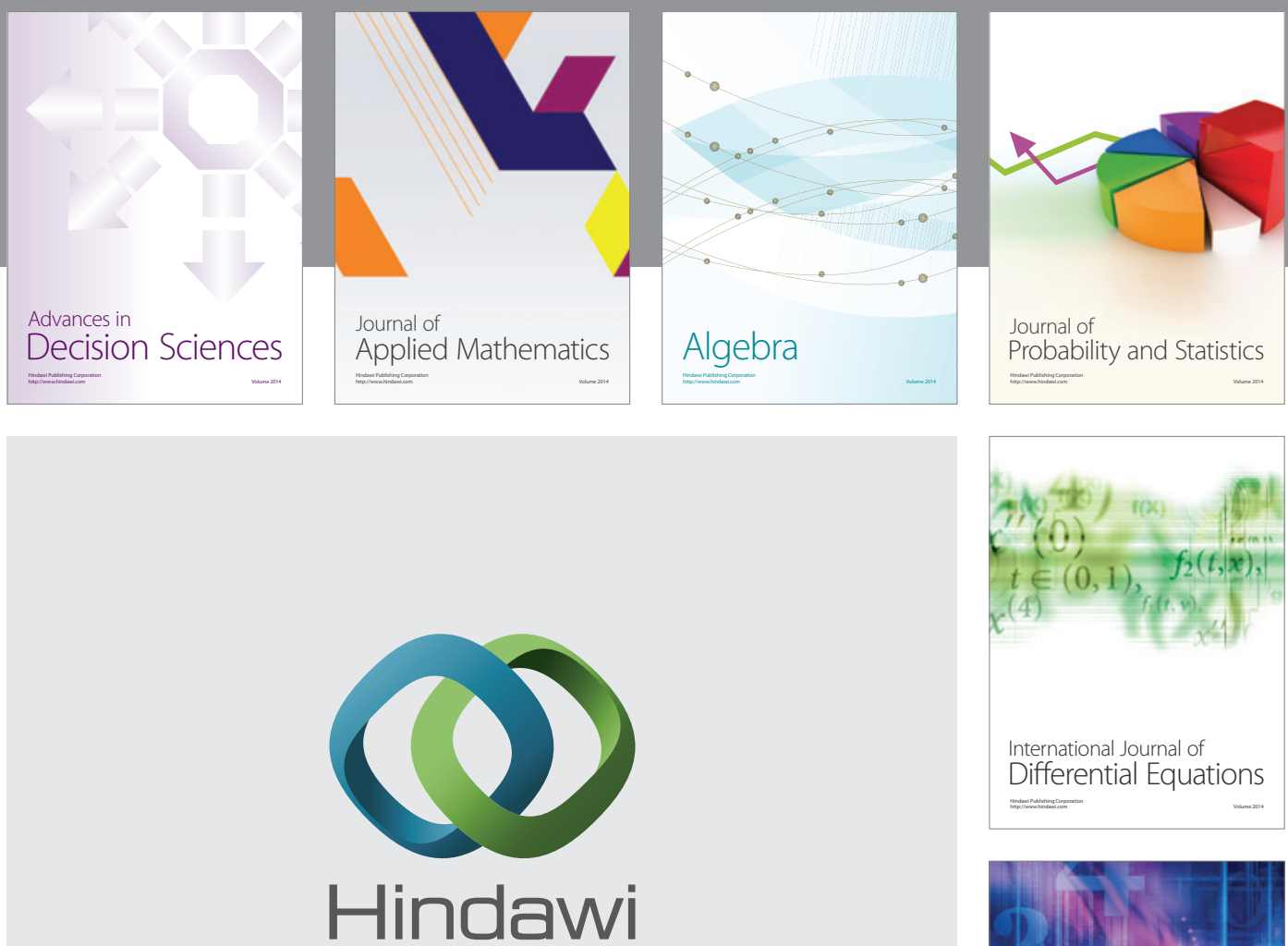

Submit your manuscripts at http://www.hindawi.com
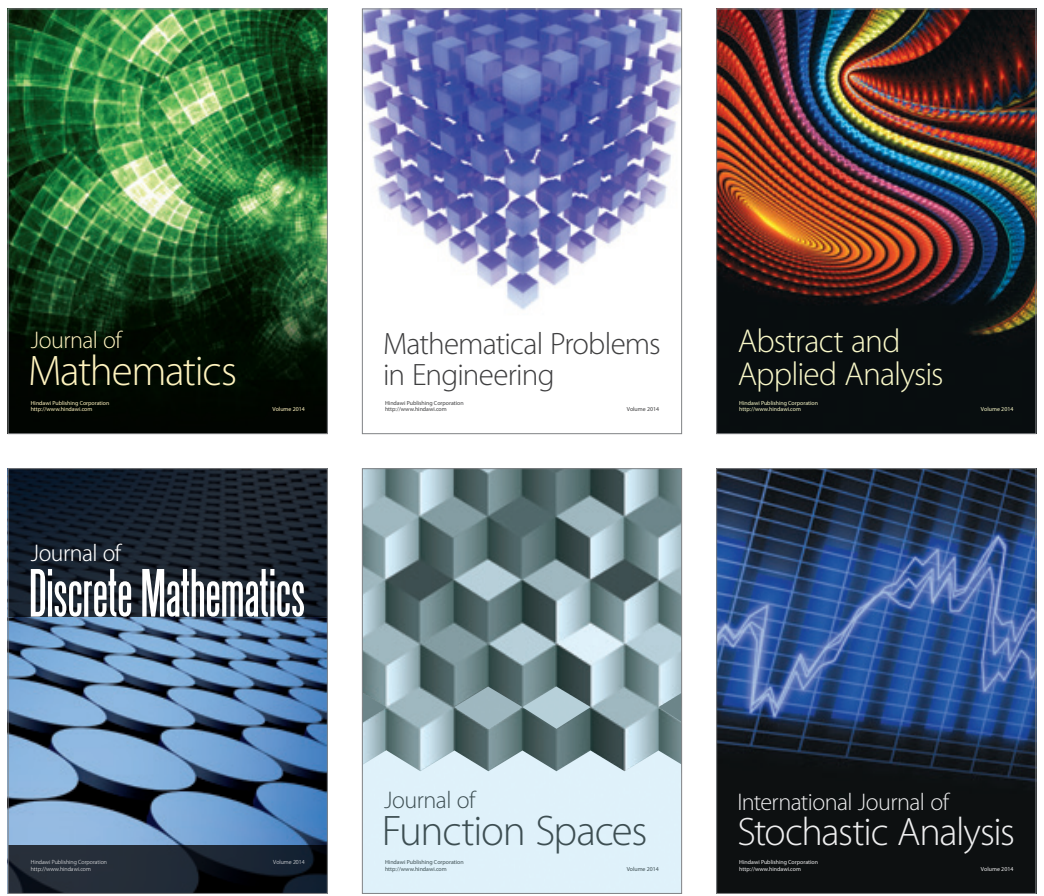

Journal of

Function Spaces

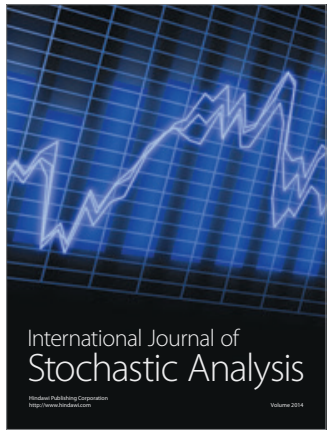

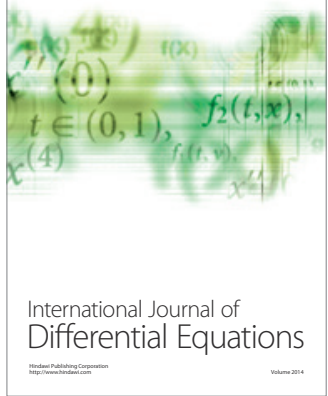
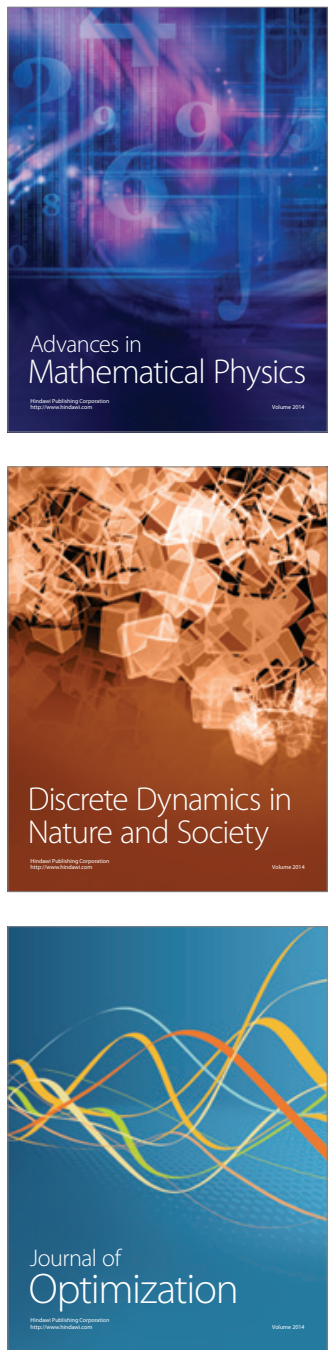